\title{
Nonlinear optical characterization of new ionic liquids by a noise reduced thermally managed EZ-Scan technique
}

Vinícius C. Ferreira, Graciane Marin, Jesum A. Fernandes, Jairton Dupont, R. B. Correia

Vinícius C. Ferreira, Graciane Marin, Jesum A. Fernandes, Jairton Dupont, R. B. Correia, "Nonlinear optical characterization of new ionic liquids by a noise reduced thermally managed EZ-Scan technique," Proc. SPIE 10684, Nonlinear Optics and its Applications 2018, 106841E (14 May 2018); doi: $10.1117 / 12.2305879$

SPIE. Event: SPIE Photonics Europe, 2018, Strasbourg, France 


\title{
Nonlinear optical characterization of new ionic liquids by a noise reduced thermally managed EZ-Scan technique
}

\author{
Vinícius C. Ferreira ${ }^{\mathrm{a}}$, Graciane Marin ${ }^{\mathrm{b}}$, Jesum A. Fernandes ${ }^{\mathrm{c}}$, Jairton Dupont ${ }^{\mathrm{b}, \mathrm{c}}$, and Ricardo \\ R. B. Correia ${ }^{a}$ \\ ${ }^{a}$ Instituto de Física, Universidade Federal do Rio Grande do Sul, Av. Bento Gonçalves 9500, \\ Porto Alegre, Brazil \\ ${ }^{\text {b}}$ Laboratório de Catálise Molecular, Instituto de Química, Universidade Federal do Rio \\ Grande do Sul, Av. Bento Gonçalves 9500, Porto Alegre, Brazil \\ ${ }^{\mathrm{c} S c h o o l}$ of Chemistry, University of Nottingham, University Park, Nottingham NG7 2RD, U.K.
}

\begin{abstract}
Thermal and nonlinear optical characteristics are evaluated for a novel ionic liquid, BMIOMe.NTf ${ }_{2}$, by means of the well established thermally managed eclipse Z-scan technique (EZ-scan). Nevertheless, this method still requires samples with good optical quality in order to provide a reasonable signal-noise ratio (SNR) when the material's nonlinearity is small. Two different procedures where then introduced to improve the SNR against sample linear inhomogeneities and scattering. Both procedures are based in a subtraction of noisy background signals from the nonlinear signal of EZ-scan output. The first is applied to thermal effects and slow sample's response, based on the perform a subtraction of the initial exposure time from the steady state signal, eliminating the linear noise. In this case, we could verify an increment of SNR from 1:2 to 40:1. The second procedure is related to fast response, e.g., arising from the electronic nonlinear polarizability. This consists in performing two sequential measurement, one with a decade of intensity higher than the first. After performing the subtraction between them, linear background suppression can be verified, with improvement of SNR to $2: 1$, amount about 10 times the signal value. Performing these procedures, we present a study of properties of ionic liquid BMIOMe. $\mathrm{NTf}_{2}$. This presents a steady cumulative effect of multiple pulse absorption leading to thermal lensing as measured by the thermally managed EZ-scan technique. The photoinduced conductivity effect observed for this ionic liquid is discussed based on the observed photothermal conversion. Also a fast response corresponding to a nonlinear refractive index was also registered when the time evolution is extrapolated for short times.
\end{abstract}

Keywords: EZ-scan, ionic liquid, nonlinear refractive index, thermal optical properties, noise suppression

\section{INTRODUCTION}

The development of new nonlinear optical materials and their characterization are essential in telecommunications, signal processing and many other technologies. ${ }^{1}$ Nonlinear refractive index and thermo-optical properties are usually the most relevant properties since they are inherent for the majority of optical materials.

There are many techniques to measure these properties of materials. ${ }^{2}$ Among them Eclipse Z-scan (EZ-scan) is one simple, direct and sensible, which measures light transmission around a circular obstacle as the result of the self-induced modification of the wave-front of a laser focused on a thin sample. ${ }^{3}$ Thereby, the signal measured from a sample scanned along the focal region determines the optically induced changes. ${ }^{3,4}$ In the particular case, when a high repetition laser is used, cumulative effects are generated by laser absorption and, consequently, the sample heating. To evaluate the corresponding optical modifications, samples must be good optical quality, scattering must be minimized from cuvette wall or samples inhomogeneity. In order to suppress

Further author information:

Vinícius C. Ferreira: E-mail: vinicius.ferreira@ufrgs.br

Ricardo R.B. Correia: E-mail: rego@if.ufrgs.br

Nonlinear Optics and its Applications 2018, edited by Benjamin J. Eggleton, Neil G. R. Broderick, Anna C. Peacock Proc. of SPIE Vol. 10684, 106841E · @ 2018 SPIE · CCC code: 0277-786X/18/\$18 · doi: 10.1117/12.2305879 
noise and increase the signal-noise ratio (SNR), we propose a very efficient procedure applied to the fast and slow response.

Ionic liquids (ILs), constitute a group of organic molten salts developed over the last decades and now introduced in many technological applications, specially because of the assigned supramolecular fluid behavior originated from the ILs extended hydrogen-bond networks. Among its distinct properties, ILs present high chemical and thermal stability, no measurable vapor pressure, a large electrochemical window (up to $7 \mathrm{~V}$ ), a variable solubility with organic compounds and biocompatibility. ${ }^{5,6}$ Beside these properties, a novel synthesized ionic liquid 1-(2-methoxy-ethyl)-3-methyl imidazolium (trifluoromethylsulfonyl)imidate, with molecular formula BMIOMe.NTf 2 , presented a modification of its electrical conductivity in the presence of the light, i.e., when illuminated with a Xe-lamp. Here we also aim to relate the optical absorption with thermal changes in this sample and establish the connection to the observed thermoelectric effect. In order to investigate this property, nonlinear optical analyzes were performed by thermal management EZ-scan technique. Analysis focused in fast response of BMIOMe.NTf 2 , i.e., distortions of wave front in EZ-scan generate by nonlinear refractive index, will also be presented and discussed.

\section{NOISE SUPPRESSION APPLIED TO THE EZ-SCAN TECHNIQUE}

Z-scan technique is a well-established method to measure nonlinear refractive index ${ }^{2}$ and thermal-optical properties of optical materials, ${ }^{4,7}$ where the self-focusing effect is evaluated by moving a sample through the focus of a laser beam. By modeling the observed variations of transmittance, it is possible to quantify optical characteristics, e.g. nonlinear refractive index and thermal-optics properties. For the Z-scan, in the far field, a circular aperture is setup in front of the detector, in contrast a disc is the spatial selector in far field to the EZ-scan technique. This different setup enhances the sensibility when compared with the Z-scan. ${ }^{3,8}$

Scanning the sample along the beam path near the focal point requires good quality of samples and its surfaces. Any interference due to multiple reflections on the walls, dirty in the cuvette or inhomogeneities decrease dramatically the SNR. To perform the optical characterization, the SNR must be improve and the experimental data correctly analyzed.

In this first section, we will introduce and discuss the subtraction of linear effects in order to attenuate noise and suppress systematic signal from the transmission data of EZ-scan technique. The procedure which will be presented here can be applied to measure fast and slow signals, e.g. electronic and thermal origin respectively, in samples which presents not excellent optical quality.

The experiment setup is based EZ-scan technique. In our system, the disc used as spatial select blocks $98.3 \%$ of beam (1.7\% of external portion of the beam arrive in the detector). The laser source was a modelocked Ti:Sapphire laser oscillator $(76 \mathrm{MHz}, 100 \mathrm{fs}, 800 \mathrm{~nm}$ ) with beam waist radius of $35 \mu \mathrm{m}$ at focal point. Additionally for the thermal response evaluation, the beam is chopped at $9 \mathrm{~Hz}$. A set of polarizer and half-wave plate is used to control the intensity input in the sample. The system is controlled by a homemade program, which controls the sample scan and acquire a temporal evolution curve of transmittance for each scan position from a oscilloscope. A scheme of the experimental setup is presented in figure 1.

High repetition lasers must be chopped in order to analyze the signal dynamics. Usually, even small absorption introduces cumulative effects leading to sample heating and, consequently, optically induced thermal effects. In this case, a thermal management approach is performed to separate the thermal contribution from electronic effects. ${ }^{9}$ The temporal window of the chopped beam is recorded and for every position of the scanned sample, the time evolution is registered in order to separate different process within the signal temporal evolution. ${ }^{7,9}$ Concerning the thermals effects, the chopper duty cycle should respect the thermal relaxation time between two exposed windows. ${ }^{7}$ Also a fast opening transition is desired to allow the measurement of fast effects. Our setup was built to provide a sample illumination (time exposure window) of $2.66 \mathrm{~ms}$ and duty cycle of $2.4 \%$ with an opening rise time of about $15 \mu \mathrm{s}$. The method here presented used these expositions time and chopped beam as strategy to enhance the SNR. 


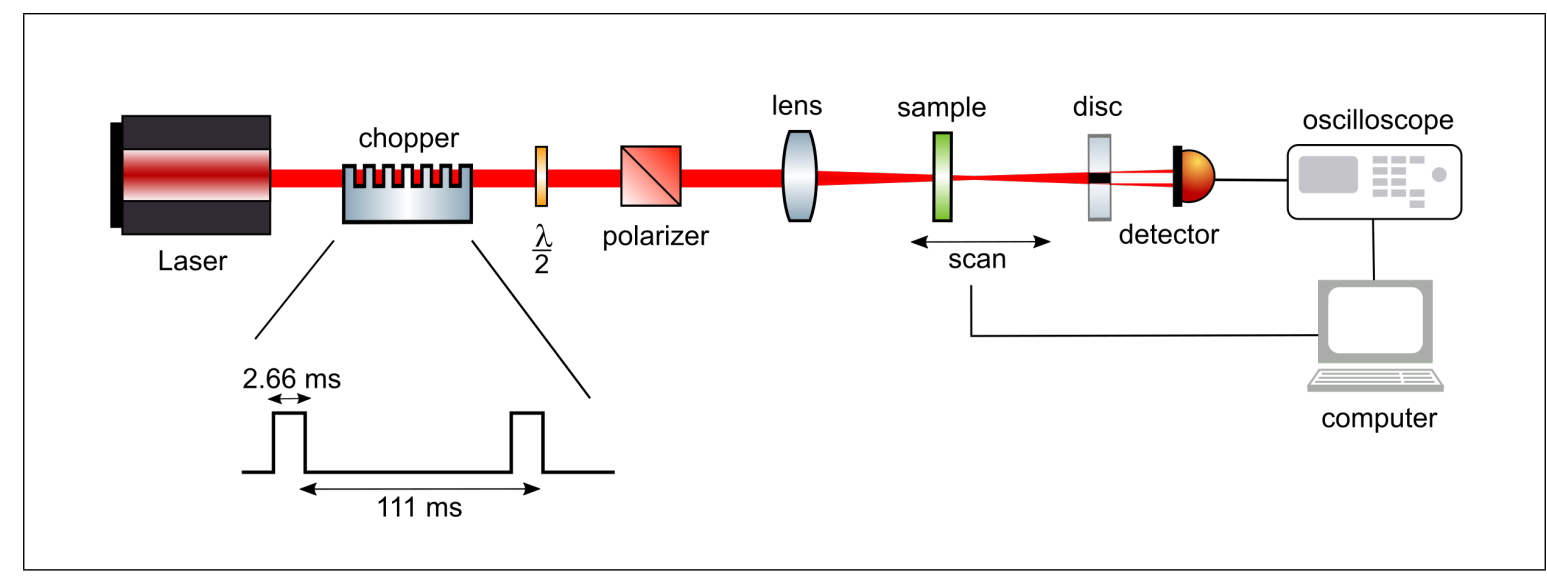

Figure 1. EZ-scan experimental setup. It consists in a chopped source laser, a set of polarizer and half-wave plate to control the intensity input, lens, sample, disc as spatial selector, detector and a computer that control the scan and save the time evolution window. Details of time exposure window of the beam are also presented.

\subsection{Noise suppression applied in the slow response}

This procedure is based on the subtraction of the normalized transmittance signal in the beginning of the chopped temporal window (typically $25 \mu \mathrm{s}$ ) from the signal at the end of this period, i.e. steady signal (typically 2.25 $m s$ ). The validity of this procedure is restricted to low laser powers and low duty cycle, in order to reduce the error introduced by the sample heating at the initial time considered. This procedure turns out as a good method to distinguish thermal effects from other optical fast responses, since the output signal is related to purely cumulative responses, without fast effects. Furthermore, this method also minimizes the noise caused by linear sample inhomogeneities (e.g., scattering from cuvette imperfections), since this noise signal usually adds up equally for both short as well as long measured times.

To exemplify this procedure of distinction, deionized water was used. The measurement was perfomed with average power equal to $100 \mathrm{~mW}$ (equivalent to $1 \mathrm{GW} / \mathrm{cm}^{2}$ ). Figure 2a presents a normalized transmittance of this sample at two distinct times, $25 \mu \mathrm{s}$ and $2.25 \mathrm{~ms}$. In this case, all time evolution curves are acquired in the same scan, comprising the similar noise amount at both times. Figure $2 \mathrm{~b}$ shows the resulting of subtraction, exhibiting a very clear thermal signal, with profile similar to theoretical reference. The noise suppression in this case was impressive. According the measurement, is possible conclude an improvement of signal noise ratio from 1:2 to 40:1. Signals from nonlinear electronic effects were not observed for deionized water in our system.

A different sample with stronger thermal effects, consisting in a solution of carbon nanodots in poly(ethylene glycol) $\mathrm{PEG}_{200 \mathrm{~N}}$, was characterized. ${ }^{4}$ This colloidal suspension has nanodots with average size of $3 \mathrm{~nm}$ and concentration of $0.0725 \mathrm{mg} / \mathrm{mL}$. Fitting experimental data is possible characterize the thermo-optical coefficient, variation of refractive index by temperature $d n / d T$. This sample presented the thermo-optical coefficient equal to $-14 \pm 1 \times 10^{-5} K^{-1}$. The experimental data to initial and final times of exposure window are presented in the figure 3a. The measurement was performed with beam average power of $9.5 \mathrm{~mW}$, which results in a beam peak power equal to $64 \mathrm{MW} / \mathrm{cm}^{2}$. Theoretical fit and the subtracted experimental data are presented in figure $3 \mathrm{~b})$. The SNR, which before the procedure was approximatelly 1:1, after the noise suppression was equal to 10:1, evidencing noise attenuating. The same characterization was performed to others two concentrations of this colloidal suspension and to $\mathrm{PEG}_{200 N} .{ }^{4}$ This sample did not present any electronic response to our system configuration.

\subsection{Noise suppression applied in the fast response}

The second method is applied to measure fast responses, e.g. nonlinear refraction index and two photon absorption. It consists basically on a input level corrected background subtraction, carrying out two sequential similar scans, differing only by the incident power. This procedure considers that strong signals presents the nonlinear effect and all linear effect (scattering and inhomogeneity) from the sample while the weak signal has no nonlinear 


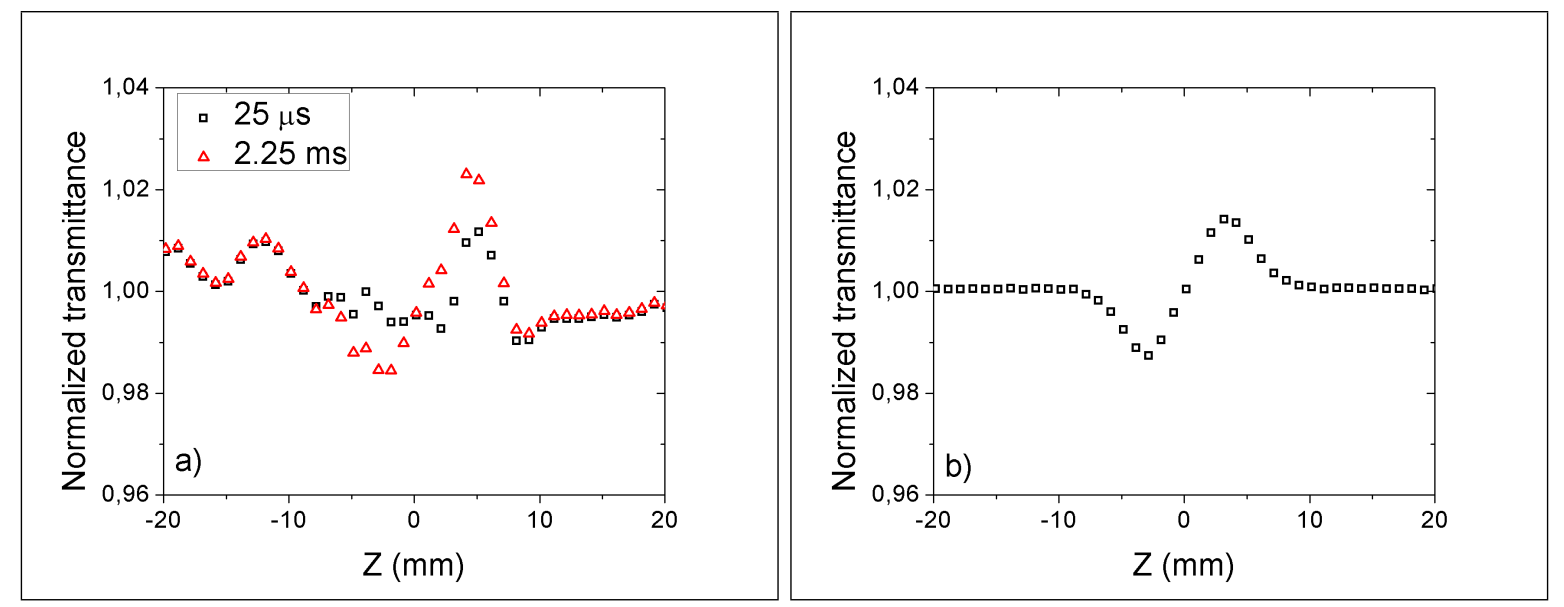

Figure 2. Normalized transmittance of deionized water, with average power equal to $100 \mathrm{~mW}$ (equivalent to $1 \mathrm{GW} / \mathrm{cm}^{2}$ ), presents a) initial and final times of the temporal window, black squared to $25 \mu \mathrm{s}$ and red triangle related to $2.25 \mathrm{~ms}$, and b) the subtracted signal.

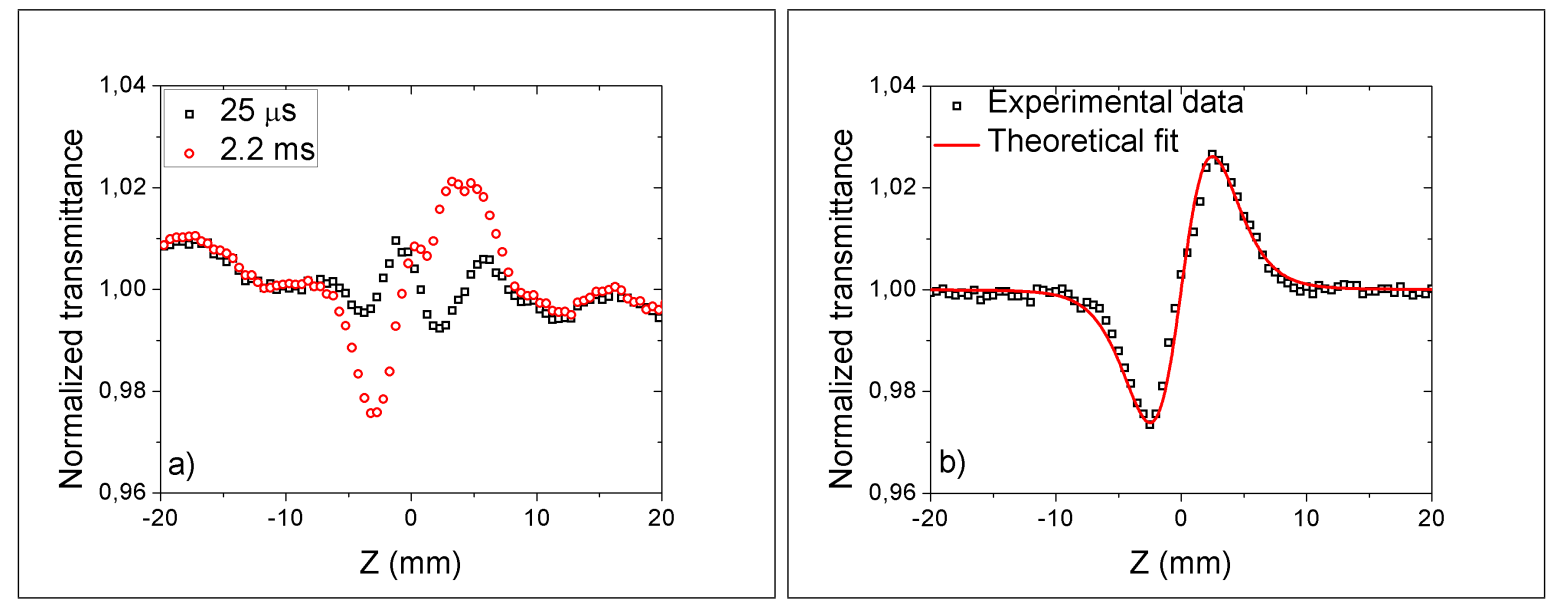

Figure 3. Normalized transmittance of carbon nanodots in $\mathrm{PEG}_{200 N}$. a) Scan at two different times, black squared and red circle for $25 \mu \mathrm{s}$ and $2.25 \mathrm{~ms}$ respectively, with laser average power equal to $9.5 \mathrm{~mW}$ (equivalent to $64 \mathrm{MW} / \mathrm{cm}^{2}$ ). Short times expositions do not present observable thermal effects while long times exposition are in a steady state of this effect. b) Subtraction between this two scans and theoretical fit. Plot adapted with permission from ref. ${ }^{4}$

effect or a negligible quantity of this. Typically, a tenth or less of nonlinear measure intensity must be used to negligence the nonlinear effects. In many cases, due to the sensitivity of the system, a tenth of intensity is the same as not distinguishing the signal from the system noise, therefore, no nonlinear signal can be evidenced in this case. Performing the corrected power subtraction between them, linear effects will vanish, revealing the nonlinear signal.

Figure 4a presents an EZ-scan measurement of a silicon carbide sample with high and low intensity, in which a noticeable interferometric pattern is apparent. This characteristic noise came from internal reflection of sample. The acquisition of both measures was performed $25 \mu \mathrm{s}$ after the chopper aperture, characterizing negligible thermal effects. Figure $4 \mathrm{~b}$ shows the results of the subtracted method to this sample, where a variation of transmittance is evidenced, confirming the presence of nonlinear response (by nonlinear index refraction) to this system. A solid guiding line is presented in order to help the curve shape visualization. This sample did not present nonlinear index absorption measured by the open Z-scan to our system.

The noise extenuation presented in silicon carbide was essential to analyze the sample. After the linear background subtraction (amounting about 10 times of the signal value), the improvement indicates a signal- 

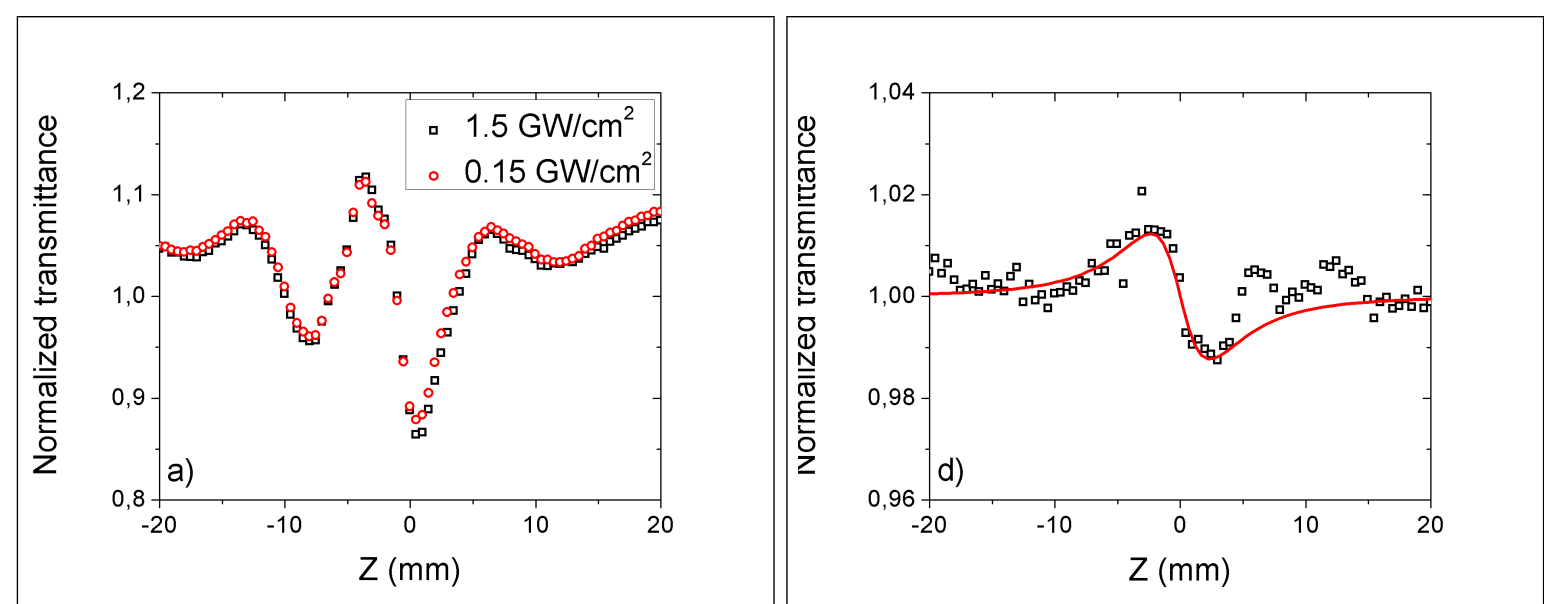

Figure 4. Normalized transmittance of silicon carbide. a) Scan of two different intensities, black squared referent of intensity equal to $1.5 \mathrm{GW} / \mathrm{cm}^{2}$ and red circles to $0.15 \mathrm{GW} / \mathrm{cm}^{2}$. b) Subtraction between two different scan with distinct intensities. Red line is a guide in order to help the visualization of normalized transmittance variation.

noise of 2:1, enough to allows the visualization of fast nonlinear effects.

\section{NONLINEAR OPTICAL CHARACTERIZATION OF IONIC LIQUID}

This section presents a study the nonlinear fast and slow response of the IL 1-(2-methoxy-ethyl)-3-methyl imidazolium (trifluoromethylsulfonyl)imidate, with molecular formula BMIOMe. $\mathrm{NTf}_{2}$. Its molecular structure are present inset of figure 5. This IL evidenced a modification of electrical conductivity upon the presence of the light. It can be explained by mechanisms causing the direct effect on carriers number, either on its number or mobility. Heating the sample by light absorption, would therefore affect the mobility. In our results, we can verify of a notable thermal modification upon a weak light absorption of these ionic liquids, which could explain the conductivity results.

Figure 5 presents the absorption spectrum of the BMIOMe.NTf2 as evaluated in a Cary 5000 UV-Vis-NIR (Agilent) with a $2.0 \mathrm{~mm}$ path length fluorescence quartz cuvette by Hellma Analytics. The sample presents a strong absorption in the near the infrared, which by means on an efficient thermal conversion would cause sample heating and consequently increase the amount of carriers.

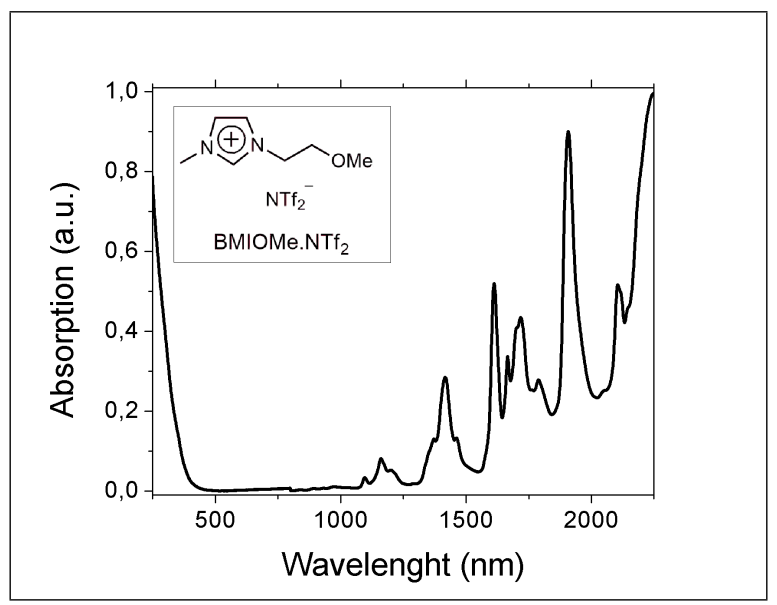

Figure 5. Spectral absorption of ionic liquid used in this work, BMIOMe. $\mathrm{NTf}_{2}$ and its molecular structure on the inset. 
In order to measure the magnitude of sample heating, EZ-scan technique was performed to BMIOMe.NTf ${ }_{2}$. Figure $6 \mathrm{a}$ presents variation of normalized transmittance to distinct times of beam exposure. These both measured were executed with power average equals to $50 \mathrm{~mW}$. The shortest time, $25 \mu \mathrm{s}$, no thermal effects are accumulate. To higher times, $2.2 \mathrm{~ms}$, thermal effects are already established. Applying the procedure presented in the first part of this work, we perform the subtraction of both signals in order to analyze a nonlinear thermal curve with low noise, which are presented in figure $6 \mathrm{~b}$. This second image also presents subtraction to another measure with average power equal to $10 \mathrm{~mW}$. Cumulative thermal effects are observed for low beam average power, of $10 \mathrm{~mW}$ and $50 \mathrm{~mW}$, acquired in steady state, i.e. $2.2 \mathrm{~ms}$ after opening the sharp chopper window. The gain of SNR to $50 \mathrm{~mW}$ measurement was, initially, 15:6 and, after the procedure, increase to 30:1.

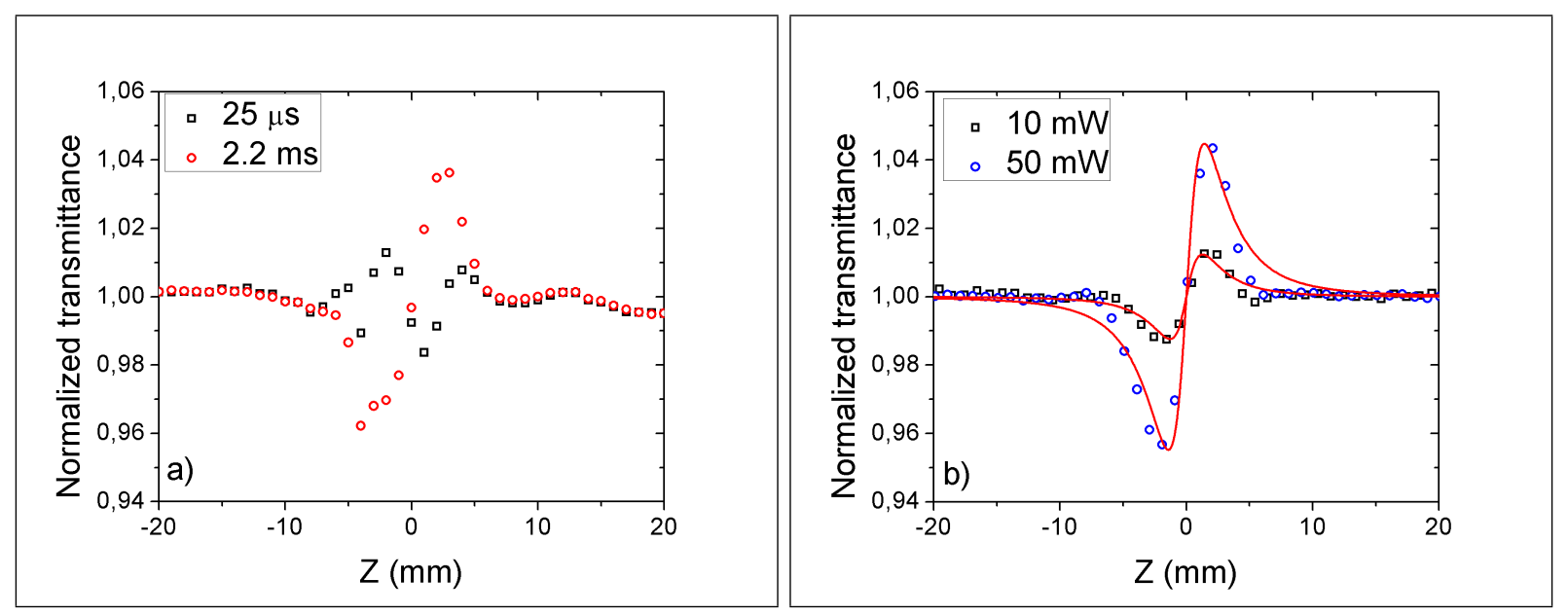

Figure 6. a) Normalized transmittance of ionic liquid BMIOMe.NTf $\mathrm{N}_{2}$ in times equal $25 \mu s$ and $2.2 \mathrm{~ms}$ with average power of $50 \mathrm{~mW}$. b) Subtraction of those signals and a result of a similar subtraction with power average of $10 \mathrm{~mW}$, blue circles and black square respectively. Red line is a guide to help the curve visualization.

Extrapolating the signal to the beginning of the beam exposure $(t=0)$, fast response related to nonlinear refractive index was also observed in this sample. Initially, the measure of IL present noise by cuvette scattering from the wall. Two sequential scans with peak intensity equal to $1.4 \mathrm{GW} / \mathrm{cm}^{2}$ and $0.5 \mathrm{GW} / \mathrm{cm}^{2}$ were performed. These are presented in 7a. After performing our procedure, a variation of transmittance could be evidenced. The curve presented in figure $7 \mathrm{~b}$ shows the variation of transmittance evaluated in times equal zero by subtracting the both sequential scans. This nonlinear effect is caused by the third order of susceptibility.

The linear background subtraction indicates a signal-noise of 3:1, about 3 times of the signal value before the procedure. As conclude in this values and in the figure 7, the observation of the electronic response of this IL would be impracticable in our system without the procedure presented here.

This sample presented a weak nonlinear noisy signal and further data are still needed for the quantitative evaluation of the nonlinearity. Open aperture scans were performed but no evidence of nonlinear absorption was observed on our system.

\section{CONCLUSION}

According our results, SNR can be improved using one of these methods based on the desired characterization. We were able to extract nonlinear signal from noisy data registered along the scan. Fast and slow materials responses could be measured following these procedures, which are easy to implement on an automated system. Table 1 presents a summary of the increase of SNR to all samples presented in this work.

Both thermal lensing and fast responses were measured for the liquid matrix of BMIOMe.NTf $\mathrm{N}_{2}$. The increase of SNR was from 15:6 to 30:1 to thermal effects. In the fast response, the IL presented a SNR of 3:1 after the procedure, amount about 3 times of the initial signal value. The phenomenon of the photoinduced change in conductivity observed in this liquid was addressed as a consequence of the heating. Derived thermal data 


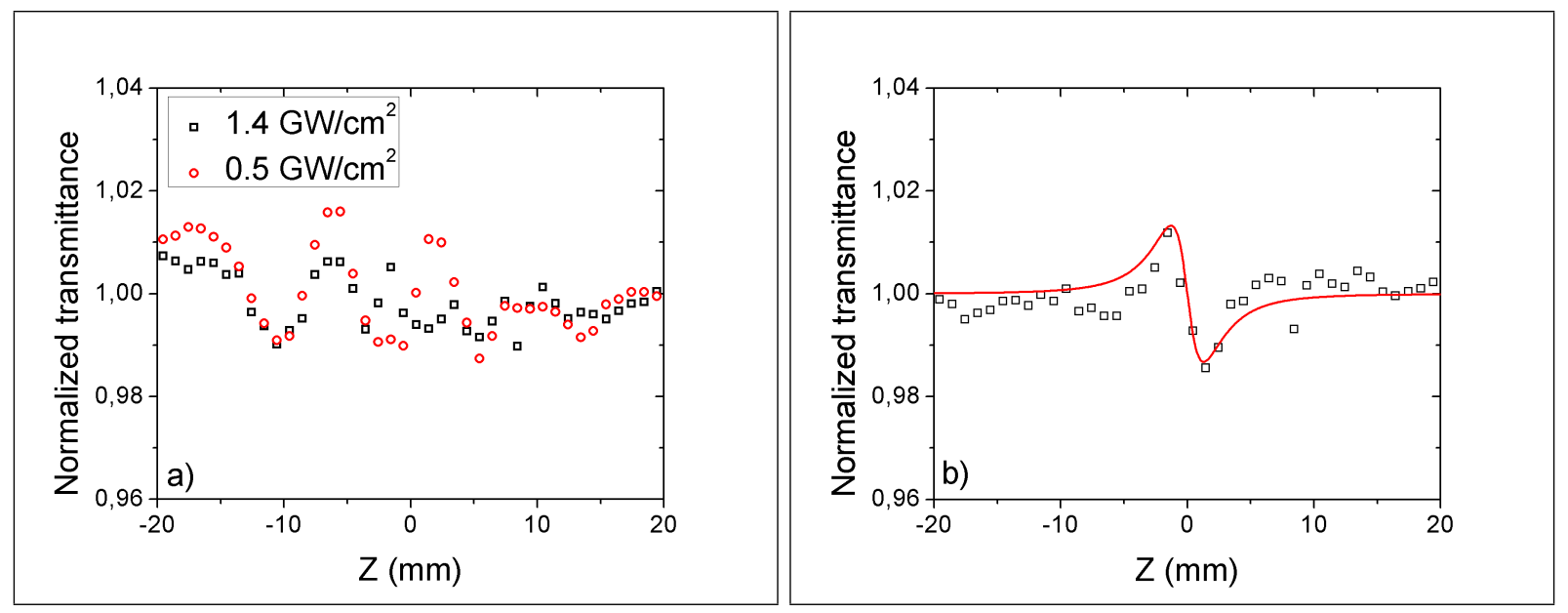

Figure 7. a) Variation of transmittance of BMIOMe.NTf $f_{2}$ to times equal zero of temporal window with high and low intensity. b) Subtraction of two different intensities evidencing the variation of transmittance and excitation of nonlinear refractive index. The solid line helps the variation of transmittance visualization.

Table 1. Summary of SNR increase to the samples used in this work.

\begin{tabular}{|l|l|l|l|}
\hline Sample & Origin of effect & SNR before the procedure & SNR after the procedure \\
\hline Deionized water & Thermal effect & $1: 2$ & $40: 1$ \\
\hline Carbon nanodots in $\mathrm{PEG}_{2} 00 \mathrm{~N}$ & Thermal effect & $1: 1$ & $10: 1$ \\
\hline Silicon carbide & Electronic effect & - & $2: 1$ \\
\hline Ionic liquid BMIOMe.NTf & \\
\hline Ionic liquid BMIOMe.NTf & Thermal effect & $15: 6$ & $30: 1$ \\
\hline
\end{tabular}

will be associated to complementary thermoelectric characterization to establish a final comparison. Nonlinear refraction index data are now being performed under a numerical careful evaluation. Similar analyses will also be performed on similar ILs presenting the same thermoelectric effect.

\section{ACKNOWLEDGMENTS}

We are grateful to $\mathrm{CNPq}$ (Brazilian National Research Program).

\section{REFERENCES}

[1] Saleh, B. E. A. and Teich, M. C., [Fundamentals of photonics], John Willey \& Sons, New Jersey, second ed. (2007).

[2] Sheik-Bahae, M., Said, A. A., Wei, T.-H., Hagan, D. J., and Stryland, E. W. V., "Sensitive measurement of optical nonlinearities using a single beam," IEEE Journal of Quantum Electronics 26, 760-769 (1990).

[3] Xia, T., Hagan, D. J., Sheik-Bahae, M., and Stryland, E. W. V., "Eclipsing z-scan measurement of $\lambda / 10^{4}$ wave-front distortion," Opt. Lett. 19, 317-319 (1994).

[4] Castro, H. P. S., Pereira, M. K., Ferreira, V. C., Hickmann, J. M., and Correia, R. R. B., "Optical characterization of carbon quantum dots in colloidal suspensions," Optical Materials Express 7, 401-408 (2017).

[5] Dupont, J. and Suarez, P. A. Z., "Physico-chemical processes in imidazolium ionic liquids," Physical Chemistry Chemical Physics 8, 2441-2452 (2006).

[6] Souza, R. F., Alencar, M. A. R. C., Meneghetti, M. R., Dupont, J., and Hickmann, J. M., "Nonlocal optical nonlinearity of ionic liquids," J. Phys.: Condens. Matter 20, 155102 (2008). 
[7] Gnoli, A., Razzari, L., and Righini, M., "Z-scan measurements using high repetition rate lasers: how to manage thermal effects," Opt. Express 13, 7976-7981 (2005).

[8] Gomes, A. S. L., Filho, E. L. F., and de Araújo, C. B., "Thermally managed eclipse z-scan," Optics Express 15, 1712-1717 (2007).

[9] Falconier, M., "Thermo-optical effects in z -scan measurements using high-repetition-rate lasers," J. Opt. A: Pure Appl. 1, 662-667 (1999). 